\title{
Establishment of Bimolecular Complementation System for the Interaction Detection between Epstein-barr Virus Nuclear Antigen 1 and Host Proteins
}

\author{
Lielian Zuo*, Meijuan Zhu*, Shujuan Du, Qijia Yan, Haibo Yu, Jing Yang, Guiyuan Li \& \\ Jianhong $\mathrm{Lu}^{\#}$ \\ Cancer Research Institute, Xiangya School of Medicine, Central South University, Changsha, P.R. China
}

Liming He

Newlife BioChemEx LLC, Bethesda, MD, USA

* These authors contributed equally to this work.

" Corresponding author

\begin{abstract}
Epstein-Barr virus nuclear antigen 1 (EBNA1) is essential for EBV episome's replication and mitotic segregation. During this process, EBNA1 may interact with a lot of host factors. Bimolecular complementation (BiMC) assay is a useful tool for a detection of protein-protein interaction in living cells. In the present study, a plasmid for expressing a fusion protein CYFP-EBNA1 was constructed. As expected, it could express CYFP-EBNA1 and localize in the cellular nuclear. The result also demonstrated that the plasmid is allowed to be used in the BiMC system.

KEYWORD: Bimolecular complementation; Epstein-Barr virus nuclear antigen 1; Protein-protein interaction
\end{abstract}

\section{INTRODUCTION}

Epstein-Barr virus (EBV) is a ubiquitous gammaherpesvirus, which is causatively associated with the development of several human malignancies, including Burkitt's lymphomas and nasopharyngeal carcinoma (NPC) [1]. Following primary infection EBV establishes lifelong persistent infection[2]. EBV replication is under the control of some host and virus factors. Epstein-Barr virus nuclear antigen 1 (EBNA1) is the only viral protein expressed in all types of tumors, and it is essential for the replication and mitotic segregation of EBV episomes to daughter cells[3]. During the viral replication and segregation, EBV may interact with a series of host proteins[4]. Bimolecular complementation (BiMC) assay is a useful approach method for the detection, visualization and map of protein-protein interactions in live mammalian cells[5]. In this system, the enhanced yellow fluorescent protein (EYFP) is split into $\mathrm{N}$ - and $\mathrm{C}$ terminal fragments, and reconstitution of these two fragments takes place by the mediation of a proteinprotein interaction, thus resulting in irreversible fluorescent signal[6]. In order to improve our understanding of the interaction patterns of EBNA1 and host proteins, in this study, we established a BiMC assay system expressing a fusion protein of EBNA1 and the C-termini of EYFP, and this system was employed in the assay for a interaction detection of EBNA1 with one host protein in living $293 \mathrm{~T}$ cells.

\section{MATERIALS AND METHODS}

\subsection{Cells, antibodies and plasmids}

Human embryonic kidney cells (293T) and COS-7 were ATCC origin and maintained in Dulbecco's modified Eagle medium (DMEM) supplemented with $10 \%$ fetal calf serum. Mouse monoclonal antibodies against c-MYC and EBNA1 were products from Sigma and Abcam companies respectively and used according to the manufacturer's instructions. The anti- $\beta$-actin antibody (Santa Cruz) was used as a loading control. Plasmid pCAGGS vector, the plasmid containing full-length Venus YFP and the plasmid pNYFP-X1 containing fused N-ternimal YFP and cellular X1 protein gene were kind gifts from Dr. Harty (University of Pennsylvania) [7]. All recombinant plasmids containing C-termini of YFP and EBNA1 gene were constructed by standard cloning techniques using the pCAGGS vector.

\subsection{Western blotting}

Western blotting were performed as described previously[8]. Protein samples were subject to electrophoresis in SDS-PAGE gels and analyzed 
under the Molucular Imager by using the Image Lab software (Bio-Rad).

\subsection{Immuno-fluorescence assay}

Immunofluorescence was performed for the detection of the expression of fused CYFP-EBNA1. Fresh COS-7 cells were seeded onto coverslips in wells of a six-well plate. At $24 \mathrm{hr}$ post-transfection of the plasmids using the TurboFect Transfection Regeat (Thermo), the cells were treated with $0.25 \%$ triton X-100 and washed with PBS, then the first antibody was added and incubated for $1.5 \mathrm{hrs}$ at room temperature. After three washes with PBS, the coveslips were incubated with the second antibodies, Alexa Fluo @ 488 monkey anti-rabbit IgG (H+L) (Life Technologies) for $1 \mathrm{~h}$ at room temperature and washed three times. Then they were treated with Hoechst (Beyotime Inc., China) for $10 \mathrm{~min}$ and washed. The coverslips were observed under a fluorecent microscope.

\subsection{BiMC assay}

Human 293T cells were grown on coverslips as described above. The pCAGGS-CYFP-EBNA1 and pCAGGS-NYFP-X1 plasmids were co-transfected into the cells. At $24 \mathrm{hr}$ post-transfection, cells were washed twice with PBS and fixed with cold methanol-acetone $(1: 1, \mathrm{v} / \mathrm{v})$ for 10 minutes at room temperature. Cells were washed and subsequently stained with Hoechst for 10 minutes at room temperature. Cells were washed and slides were viewed under a fluorescent microscope.

\section{RESULTS AND DISCUSSION}

\subsection{Construction of plasmid pCAGGS-CYFP- EBNA1}

The basic strategy of BiMC is illustrated in Figure 1.
A
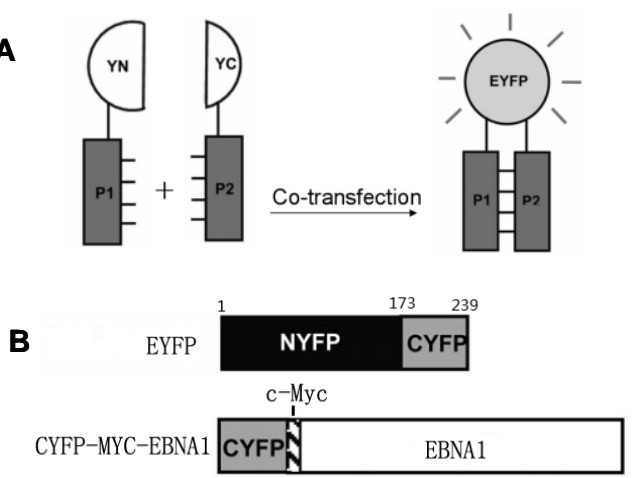

Figure 1 Schematic diagram of EYFP fusion proteins. (A)Diagram of the basic principle of BiMC assay. EYFP is split into $\mathrm{N}$ - and $\mathrm{C}$ - tag terminal fragments ( $\mathrm{YN}$ and $\mathrm{YC}$ ), which are joined in-frame to 2 proteins (P1 and $\mathrm{P} 2)$. Interaction of $\mathrm{P} 1$ and $\mathrm{P} 2$ leads to reconstitution of EYFP with a fluorescent signal. (B)The structure of CYFP-EBNA1. The C-terminal of EYFP (aa 193-239) was joined in-frame to EBNA1 with a cmyc tag as indicated.
The C-terminal of EYFP was firstly cloned into pCAGGS vector, resulting in a plasmid designated pCAAGS-CYFP. EBNA1 gene was also PCRamplified from the EBV B95.8 strain. A c-myc tag at the $\mathrm{N}$-terminal was introduced by the forward primer, and was cloned into pCAGGS-CYFP, resulting in a fused CYFP-cMyc-EBNA1 expression box. The expression plasmid was identified by using restriction enzymes according to the design and sent for being sequenced, and the result showed that the plasmid was successfully constructed.

\subsection{Expression of EBNA1 detected by western blotting assay}

In order to make sure that the expression plasmid was able to express fused CYFP-cMYC-EBNA1 as expected, the plasmid was transfected into 293T cells which is EBV negative, at $24 \mathrm{hr}$ posttransfection, the general cellular protein was extracted and subject to a western-blotting assay. As Figure 2 showed, both anti-cMyc and anti-EBNA1 antibodies could detect the expression of the expected fusion protein with an expected size of about $70 \mathrm{kD}$.

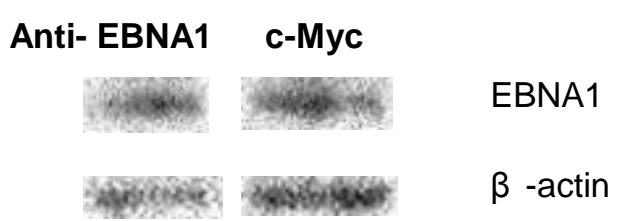

Figure 2 The detection of fused CYFP-MYC-EBNA1 by western blotting

\subsection{Localization of CYFP-EBNA1 in living cells}

EBNA1 is important for the replication and mitotic segregation of EBV episomes, and usually expressed in cellular nuclear. Here, immunofluorescence assay, in which anti-cMyc antibody was used as specific antibody, was employed to detect if fused CYFPEBNA1 also localization in the nuclear of COS-7 cells. As it was shown in Figure 3, the fusion protein CYFP-cMYC-EBNA1could localize in the nuclear, with the minority being expressed in cytoplasm.

\subsection{Interaction assay of EBNA1 and host protein by BiMC}

Some host proteins were reported to interact with EBNA1 during the replication and segregation of EBV episomes. In this study, we tried one since we speculated it may also be involved in the interaction. The result also showed that the interaction took place in the nuclear (Figure 4). 
Figure 3. Localization of the fusion protein CYFP-cMYC-EBNA1 in COS-7 cells

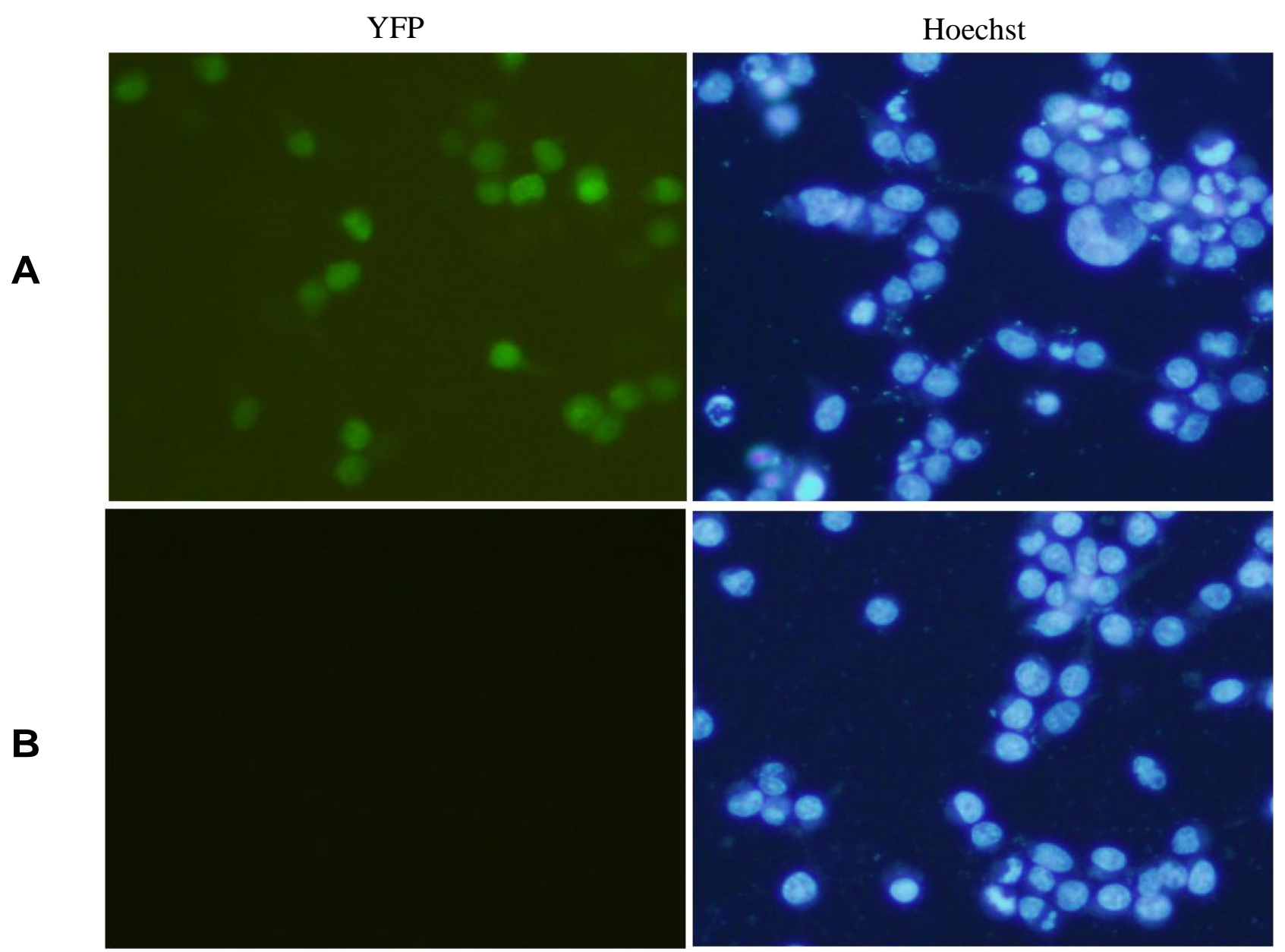

Figure 4 Interaction assay of EBNA1 and host protein by BiMC (A) Co-transfection of pCAGGS-CYFP-EBNA1 and pCAGGSNYFP-X1. (B)Transfection of only pCAGGS-CYFP-EBNA1.

In conclusion, we have successfully established the BiMC assay system for the interaction detection of EBNA1 with host proteins. BiMC approach is useful for detecting and recording transient interaction events, allowing for detection of shortlived and weak protein-protein interactions in intact living cells. The outstanding advantage of this assay is that it enables characterization of protein-protein interactions in the natural cellular environment[5-7]. The establishment of this system in the present study would largely help us to understand the interaction pattern of EBNA1 with host proteins, and provided the feasibility of using the BiMC approach for our further understanding about the roles of EBNA1. 


\section{ACKNOWLEDGEMENTS}

This work was supported by the projects of National Natural Science Foundation of China (81171931, 81372139) and Hunan Provincial Natural Science Foundation of China (2015JJ2149).

\section{REFERENCES}

[1] Young LS, Rickinson AB. Epstein-Barr virus: 40 years on. Nat Rev Cancer 2004, 4:757 -768.

[2] Young LS, Murray PG. Epstein-Barr virus and oncogenesis: from latent genes to tumours. Oncogene 2003, 22:5108-5121.

[3] Frappier L. EBNA1 and host factors in Epstein-Barr virus latent DNA replication.
[4] Frappier L. The Epstein-Barr virus EBNA1 protein. Scientifica 2012, 2012:438204.

[5] Kerppola TK. Visualization of molecular interactions by fluorescence complementation. Nat Rev Mol Cell Biol 2006, 7:449-456.

[6] Kerppola TK. Bimolecular fluorescence complementation: visualization of molecular interactions in living cells. Methods Cell Biol 2008, 85:431-470.

[7] Liu Y, Stone S, Harty RN. Characterization of filovirus protein-protein interactions in mammalian cells using bimolecular complementation. J Infect Dis 2011, 204(Suppl 3):S817-824.

[8] Lu JH, Tang YL, Yu HB, Zhou JH, Fu CY, Zeng X, Yin HL, Wu MH, Yu ZY, Zhang JY, Li XL, Li GY. EpsteinBarr virus infection facilitates the malignant potential of immortalized human epithelial cells: from latent genome to viral production and maintenance. Lab Invest. 2010 90(2):196-209. 